\title{
La construcción teórica del campo de la enseñanza deportiva desde la Globalización
}

The theoretical construction of the sports education field since Globalization

\author{
Germán Hours \\ Facultad de Humanidades y Ciencias de la Educación, \\ Universidad Nacional de La Plata, Argentina \\ gerhours22@gmail.com
}

\begin{abstract}
RESUMEN:
Este trabajo es producto del proyecto de investigación: Los discursos de la enseñanza de los deportes desde la perspectiva de la educación fisica. Análisis sobre la construcción teórica en el campo desde el comienzo de la Globalización. Proyecto en el que se indagó de manera panorámica y epistemológica sobre la conformación de los discursos que se presentan en el campo de la enseñanza deportiva, en especial, a partir del comienzo de lo que se conoce como Globalización. Este trabajo forma parte del informe final que se presentó ante las autoridades correspondientes que evalúan y califican los proyectos de investigación en la Argentina. En este artículo se expone la manera en que esta lógica que emergió como un cambio político-comunicacional, llegó a condicionar también el campo de las prácticas deportivas y de su enseñanza.
\end{abstract}

Palabras Clave: Discursos, Deportes, Globalización, Neurociencias, Neoliberalismo.

\section{Abstract:}

This work is a product of the research project "The discourses of sports teaching from the perspective of Physical Education. Analysis of the theoretical construction in the field since the beginning of Globalization." This project investigated in a panoramic and epistemological way the conformation of the discourses that are presented in the field of sports education, especially from the beginning of what is known as Globalization. This work is part of the final report that was presented to the corresponding authorities that evaluate and qualify the research projects in Argentina. This article presents the way in which this logic that emerged as a political-communicational change also conditioned the field of sports practices and their teaching.

KeYwORDS: Speeches, Sports, Globalization, Neurosciences, Neoliberalism.

\section{INTRODUCCIÓN}

Para comenzar, se debe señalar que este trabajo es producto de la investigación que se desarrolló en el Proyecto Promocional de Investigación y Desarrollo [PPID]: Los discursos de la enseñanza de los deportes desde la perspectiva de la educación física. Análisis sobre la construcción teórica en el campo desde el comienzo de la Globalización, durante los años 2018 y 2019. ${ }^{1}$ Proyecto en el que se indagó de manera panorámica y con un carácter epistemológico sobre la conformación de los discursos que se presentan en el campo de la enseñanza deportiva, en especial, a partir de lo que se conoce como la Globalización. Investigación que de manera particular, por los indicios que fue encontrando, indagó en los vínculos establecidos entre las llamadas Neurociencias y las premisas neoliberales. En este orden, es necesario señalar que, como ya se ha establecido en numerosos estudios, la Globalización determinó un cambio en las formas de comunicación que involucró todas las dimensiones sociales, inclusive, la educativa, y en este contexto, la Educación Física y la enseñanza deportiva no fueron la excepción. Como es sabido, implicó el desarrollo tecnológico de la comunicación, posibilitando la interdependencia entre los distintos países del mundo, uniendo sus mercados, sus sociedades y hasta sus culturas, a través de una serie de transformaciones, fundamentalmente económicas y políticas, que por lo tanto poseen un carácter global.

\section{Recepción: 25 Mayo 2020 | Aprobación: 20 Septiembre 2020 | Publicación: 30 Septiembre 2020}


En el campo de la investigación en Educación Física en la UNLP, muchos proyectos ya han demostrado que no es posible elaborar una teoría de la educación del cuerpo si se piensa a esta disciplina con las herramientas de las ciencias naturales. Estas investigaciones además, han demostrado que la humanidad se ha construido sobre un conjunto de supuestos que se han tornado, tanto dogmáticos como incuestionables y que, por lo tanto, por definición no pueden ser juzgados, mucho menos cuestionados. El campo de la enseñanza deportiva, en términos teóricos, a pesar de configurarse desde algunas lógicas específicas, no es la excepción a lo que ocurre en el campo educativo y en la arena política occidental en general. Lógicas que, articulando entre lo biológico y los modos capitalistas, operan en nombre del desarrollo de la ciencia y del hombre. Lógicas que, de manera muy sutil, han generado cambios en función de establecer logros más acentuados y en un menor tiempo de ejecución. De esta manera, al analizar la conformación teórica del campo, se puede observar lo señalado por Wullian Mendoza Gil (2016), quien afirma que:

“El deporte [...] en el contexto de la globalización es una empresa cuya función es la reproducción de relaciones ideológicas, políticas y económicas del sistema social imperante, el capitalismo, que ha marcado su génesis, dinámica y estatus actual de desarrollo. Si la globalización del deporte se ha de entender más allá de una simple descripción somera, se le debe hacer en el marco de los cambios institucionales que ha sufrido producto de la mundialización y homogeneización de la sociedad que pretende por continuidad y discontinuidad histórica plantearse hegemónicamente como la única valedera" (2016, s/p).

En síntesis, en nombre del progreso se han desarrollado mecanismos que han alcanzado niveles cada vez más especializados, revistiendo de una supuesta verdad científica y de cierta naturalidad todas las prácticas humanas, en especial en aquellos discursos que provienen de las Neurociencias. En esta investigación, se partió del supuesto de que, en nombre del progreso y de la ciencia, y a partir fundamentalmente de las Neurociencias, que lograron sintetizar la idea de lo biológico y el avance científico en su accionar, el desarrollo de la enseñanza deportiva buscó acelerar los mecanismos para el triunfo, dejando como consecuencia una borrosa imagen sobre las particularidades del sujeto, rediseñándolo de manera general y totalizada en función de los intereses que cada práctica persigue.

\section{RECORRIDO METODOLÓGico}

La metodología con la que se abordó esta investigación respondió al enfoque cualitativo e interpretativo. En términos generales, en esta investigación se efectuó, en primer término, en un rastreo de los diversos discursos teóricos, para luego de diversos análisis epistemológicos, se pudiera objetivar conclusiones respecto de las formas de enseñanza que estas teorías plantean, estableciendo un análisis tanto epistémico como por momentos arqueológico. Se trató de averiguar, en definitiva, cómo se propone la enseñanza de los deportes para poder rastrear los principios que las orientan, la ideología que transmiten de manera explícita e implícita y las formas de producción de conocimiento que éstas suponen. En este sentido, es necesario resaltar la relevancia que, por ser una investigación de carácter cualitativo, que pertenece al paradigma hermenéutico, se pretendió revelar los datos de sentido, es decir, el significado que tienen los fenómenos investigados en la sociedad y como ellos condicionan al sistema educativo.

El plan de trabajo para el desarrollo del proyecto incluyó los siguientes momentos y tareas:

1. Un primer momento de la investigación, la primera mitad del primer año, se reservó para la definición y ajuste del marco teórico y metodológico de la investigación. Básicamente, se propuso una metodología de investigación de carácter exploratoria, estableciéndose ésta, en una etapa fundamental para la recopilación, fichaje y análisis de fuentes documentales, comprendidas entre investigaciones, libros, revistas, artículos periodísticos y documentos oficiales del ámbito educativo y de gobierno, y el reconocimiento de diferentes ámbitos educativos, con el objetivo de que se pudiesen identificar los discursos que se encuentran en cada uno de ellos. La idea de este momento, además de concentrar los insumos teóricos necesarios para el desarrollo 
de la investigación, fue también establecer una base de datos disponibles ante posibles necesidades en la tarea investigativa.

2. Un segundo momento de la investigación, la segunda mitad del primer año, se concentró en el análisis y la categorización de las fuentes relevadas. Básicamente se trató de una etapa metodológica destinada al proceso de categorización de los datos construidos que servirían para la posterior teorización. En este momento se significaron los datos, reduciéndolos para poder expresarlos y describirlos de manera conceptual. Esta reducción de datos, es decir, la categorización, fue considerada una operación indispensable que se realizó en el proceso de investigación cualitativa, porque posibilita la construcción teórica que se llevó a cabo en la etapa final del proyecto, considerándosela un momento clave del proceso y del proyecto.

3. El tercer momento de la investigación, la primera mitad del segundo año, se utilizó para la triangulación, teorización y producción científica y académica, sobre lo trabajado hasta ese momento. Este momento fue proyectado fundamentalmente como una etapa de ajustes finales para la teorización final sobre el objeto de investigación. Este momento, además de producir saberes para su difusión, permitió comenzar a establecer las conclusiones finales de la investigación, las que permitieron a su vez, construir las bases teóricas para el informe final y las posibilidades de una publicación más amplia como un libro u otras obras como por ejemplo este artículo. En este momento se desarrollaron de grupos de discusión con otros proyectos de investigación de ésta y otras unidades ejecutoras, como por ejemplo con el Grupo de Estudios Epistemológicos del Deporte [GEED], o en el espacio de Triangulación de Proyectos en las " $3^{\circ}$ Jornadas de Investigación Cuerpo, Arte y Comunicación. Recuperación de Prácticas y Producción de Conocimientos”, organizadas por el Área de Estudios e Investigaciones en Educación Física [AEIEF], del Instituto de Investigaciones en Humanidades y Ciencias Sociales [CONICET-UNLP], de la Facultad de Humanidades y Ciencias de la Educación, desarrolladas entre los días 6 y 7 de noviembre de 2018. En este sentido, hemos considerado para el desarrollo del proyecto, que los espacios de debate poseen un valor fundamental para ejercer la vigilancia epistemológica necesaria que requiere la construcción de conocimientos científicos.

4. El cuarto momento de la investigación, la segunda mitad del segundo año, se configuró exclusivamente para la confección de los informes finales del proyecto y posibles publicaciones, con atención también, a vincular los conocimientos producidos en este proyecto con las carreras de grado y posgrado en Educación Física de nuestra facultad, con otras instituciones educativas y con otras áreas de saberes o disciplinas educativas. Este fue un momento clave también para la difusión y divulgación de las construcciones teóricas producidas, siendo la etapa en la que más se presentaron trabajos en eventos científicos. En este momento parte de nuestra idea fue someter a una revisión real y formal los supuestos que nuestro tratamiento permitieron elaborar, tratando de alejarnos de posibles subjetivaciones que estorbaran la tarea científica, como así también, establecer límites a los dogmas y los conceptos antojadizos que pudieran provenir de nuestras propias pre-nociones.

En relación con el trabajo metodológico, específicamente relacionado con el rastreo bibligráfico, se puede señalar que, además de algunos de los textos citados anteriormente, entre las fuentes relevadas para la investigación, se han encontrado otros trabajos inherentes al objeto abordado que han sido de sumo interés, como el de la revista ALESDE, que tiene un número especialmente dedicado a este tema en 2018; el texto de Eric Dunning (2009) Reflexiones sociológicas figurativas y de proceso sobre el deporte y la globalización: algunas observaciones conceptuales y teóricas, con especial referencia al fútbol aparecido en la revista Apunts Educación Física y Deportes; el trabajo de Lara (2019) expresado en el artículo Educação Física e Cultura na América Latina; el texto de Alejandro Almonacid (2012) La educación física como espacio de transformación social y educativa: perspectivas desde los imaginarios sociales y la ciencia de la motricidad humana presentado en la revista Estudios Pedagógicos; lo expuesto por Silvana Dos Santos, Elaine Crestani, Juliana Montenegro y Larissa Lara (2015) Dimensiones de la educación física escolar colombiana e incursiones por la tematización de la cultura y la calidad en la educación, que apareció en la revista de Investigación Cuerpo, Cultura y Movimiento de Bogotá, Ediciones USTA; o el capítulo de Liliana Rocha Bidegain (2017) Educación física o educación 
corporal. Biologismo, nibilismo, individualismo, y el proyecto de investigación vigente, dirigido por esta misma autora: Prácticas corporales y educación del cuerpo: el discurso neurocientifico como imposible para la enseñanza, radicado en la Facultad de Humanidades y Ciencias de la Educación de la UNLP. ${ }^{2}$

\section{Resultados}

Para hablar de los resultados obtenidos, como primera medida se deben aclarar dos aspectos que se relacionan con los objetivos planteados en el proyecto: en primer lugar, por tratarse de una investigación cualitativa, en la que los datos estadísticos no son tan relevantes como las prácticas discursivas, nos corrimos de cualquier relevamiento cuantitativo, a excepción de aquellos que demuestren las tendencias teóricas dominantes, las cuales nuestra investigación demuestra que están a la vista; en segundo lugar, como hemos decidido plantear una investigación teórico-hermenéutica, valoramos más los enunciados que pueden ser determinantes para la conformación del sujeto, por sobre los que suponen un individuo biológicamente pre-concebido. De esta forma, queremos señalar que nuestros resultados, que han sido muy relevantes, no son configurados como suele hacerse en el campo de la investigación deportiva, sino que su valor está al otorgarle significación a los discursos para la conformación del sujeto y las tendencias dominantes en el campo, que sin dudas ha alcanzado al campo educativo de manera definitoria.

Como síntesis y conclusión, la investigación desarrollada permite afirmar que ningún discurso es más funcional al pensamiento neoliberal que el neurocientífico. En parte porque en él se propone la posibilidad en el ser humano como una pura potencia que depende de la estimulación orgánica adecuada; en parte porque construye la idea de que todos parten desde el mismo lugar, al considerar a todos como iguales. El ser humano susceptible a esas cualidades se ve inmerso en un universo teórico lleno de posibilidades, pero que, sin comprenderlo de verdad, lo termina limitando a un camino establecido por otros con fines económicos y políticos particulares. Aturdiendo al sujeto con promesas que pueden ser alcanzadas como producto de su esfuerzo, logra operar para destacar a los más dotados, dejando en el camino a los menos aptos, pero haciendo que estos últimos se hagan cargo de su incapacidad, aceptando y conformándose con los designios que un supuesto destino definió naturalmente y que los postulados médicos se han encargado de visibilizar para el bien común. Nora Merlin lo ha expresado muy claramente, al afirmar que:

\footnotetext{
"La subjetividad neoliberal se configura siguiendo el modelo empresarial planteado como una serie uniformada, en la que lo humano se reduce a su mínima expresión: todo debe estar calculado, disciplinado y controlado. Las personas se someten a los mensajes comunicacionales, que terminan funcionando inconscientemente como órdenes. De esta forma, incorporan los imperativos de la época y sustentan la creencia de que eligen libremente mensajes comunicacionales, mientras que en verdad son impuestos a fuerza de repetición y técnicas de venta" (Merlin, 2017, s/p).
}

Los deportes, que siempre han sido prácticas vinculadas al capitalismo, con el aporte de las Neurociencias, a partir de la Globalización, logaron alcances inconmensurables en la sociedad. El discurso neurocientífico que gobierna hoy las prácticas deportivas, como señala Merlin, coloniza las diversas subjetividades, apelando a la [con]ciencia moderna, que en nombre del bienestar general, disimula y convierte intereses económicos y políticos, en conocimientos neutros que se instituyen como verdades indiscutibles penetrando al sujeto y condicionándolo a un futuro pre-establecido que poco tiene que ver con sus intereses y sus decisiones. Por debajo de un conjunto de supuestos y prescripciones que configuran las razones de la existencia humana, "debe considerarse que la investigación sobre el cerebro puede funcionar como una renovada oferta de espejitos de colores. [...] Las Neurociencias, funcionales al neoliberalismo, se proponen fabricar la construcción biopolítica de un sujeto adaptado al circuito neuronal, portador de amores calculados y angustias medicadas en nombre de una supuesta salud mental equilibrada que viene con receta y protocolo." (Merlin, 2017, s/p) 
En esta investigación se encontraron gran cantidad de textos que permitieron corroborar la hipótesis de que, si bien la Educación Física permanentemente señala la necesidad de valorar la cuestión social, describiendo la existencia de dos tipos de dimensiones, una más vinculado a lo social como es la pedagógica, y otro más a las ciencias biológicas, como es el discurso del deporte salud, son las Neurociencias las que gobiernan el campo; ellas no hacen más que determinar también al campo de lo social, construyendo una teoría que sintetiza el pensamiento neoliberal a partir del concepto de calidad de vida. En definitiva, el aporte de las Neurociencias al campo de la enseñanza deportiva, fue consolidar la relación directa con el mundo económico y productivo, al reforzar la cuestión del funcionamiento del ser humano,

Como parte de los resultados, debemos señalar que, algunos de los conocimientos que se produjeron en este proyecto de investigación fueron transferidos directamente a otros proyectos de investigación -actualmente en curso- y en otros casos, de momento, sirven como insumos teóricos para la formación de grado en Educación Física de nuestra institución. En algunos casos también posibilitaron el diseño y abordajes teóricos de seminarios de posgrado y usos en otras carreras de Formación Docente como es el caso por ejemplo de algunas materias del Profesorado en Educación Física de la Universidad Provincial de Córdoba.

Del mismo modo, los conocimientos derivados del presente proyecto, tienen por objeto también, vincular los conocimientos construidos directamente con los debates al interior de la institución dedicada al desarrollo de la Educación Física, a la cual pertenecíamos al momento de esta investigación, fortaleciendo de esta manera la investigación y la producción de saberes a nivel institucional, como así también, para posibilitar debates en torno a los diversos objetos de estudio que posee la disciplina, en relación al campo teórico del deporte, pero de manera más amplia, a las prácticas corporales de manera general, por ser la Globalización un fenómeno que alcanza a todos los estamentos de las sociedades modernas occidentales.

\section{Discusión}

En el campo educativo, las nuevas tendencias aseguran de manera crítica que, adentrado el siglo XXI, "la educación se relaciona todavía con la línea filosófica del positivismo y el método científico en franca contradicción con el desarrollo de lo que llaman los expertos 'la revolución biotecnológica" (Vélez de Fonnegra, 2011, p. 1). En este sentido, siguiendo a esta autora, se puede afirmar que, en lo que refiere a explicar la conducta humana, cuestión fundamental y eje vertebrador de muchos discursos que se encuentran en el campo deportivo, la llegada de la Globalización, implicó un paulatino pero incesante resurgimiento de las Neurociencias. Por lo tanto, las indagaciones llevadas a cabo en nuestra investigación permiten señalar que, a partir del comienzo de este momento histórico y bajo este discurso médico particular, se estableció de manera más drástica al individualismo como fenómeno económico, político, social y hasta educativo. Individualismo que aparece con la misma emergencia de la Era Moderna, pero que, es necesario aclarar, alcanza su máxima conformación y expresión a partir de este momento histórico simbolizado por la caída del Muro de Berlín, que simboliza el límite temporal y significativo del objeto de estudio de esta investigación. Concebido como el convocado para acrecentar los límites de posibilidades del ser humano, el individualismo estableció un claro dominio teórico. Bajo esta impronta, la individualidad se constituyó como un método de interpretación a tener en cuenta por todos los ciudadanos en dos sentidos: como un manual utilizable en la práctica cotidiana y como un tratado de alcance teórico sobre la validez de los procedimientos interpretativos e instituyentes. Este proceso originado en la civilización occidental y que se ha expandido de manera exponencial alrededor del mundo en las últimas décadas, como es de suponerse, también alcanzó al campo deportivo. En este marco, se puede afirmar a partir de la revisión teórica del campo, que el discurso académico, sobre todo aquel proveniente de la Educación Física, que retoma las bases del discurso médico, ha favorecido las posturas que interpretan la cultura deportiva, como parte del cientificismo y economicismo dominante que ha desarrollado al deporte, en especial en las últimas décadas. Discursos que de manera abstracta refieren a un sujeto totalizado, muchas veces pensado como un individuo que referencia su lado animal, muchas otras a 
un ciudadano común, en el que hay que generar estímulos para su acceso a la práctica deportiva, puesto que éste es presentado como una necesidad para su vida, articulando esta idea con su lado natural y su capacidad de movimiento como una cualidad suprema.

Con el advenimiento de la Globalización, las Neurociencias cobraron un nuevo valor en la cultura occidental. Los avances que se han ido gestando dentro de las ciencias y, con ellas, en el campo deportivo, "llevaron a la neurociencia a constituirse en la poderosa herramienta de explicación y divulgación de la naturaleza biológica y social que posee el ser humano" (Parra Bolaños; Herrera Cárdenas y Muñoz Giraldo, 2017, p. 19). En este orden, y siendo una de las cuestiones más relevantes que se ha podido definir en esta investigación, es que a partir de la Globalización, las formas de pensar tanto la práctica como la enseñanza deportiva establecieron un claro corrimiento desde la perspectiva técnica hacia una mirada más táctica o "situacional", pero que requiere un refinamiento y una exaltación de la técnica inusitados hasta este momento histórico. Es así que la inmensa mayoría de textos que se han analizado en este campo, demuestran una mayor inclinación hacia la matriz productiva en el orden deportivo. El éxito es la guía que orienta toda práctica de enseñanza y todo discurso que se pronuncie en esa materia. La llegada del neoliberalismo implicó un cambio no sólo en el contenido de la bibliografía al respecto, sino también en el origen de la misma, para establecer una supremacía de textos provenientes de España o de países influenciados por éste, en el que casi exclusivamente se habla de iniciación deportiva.

$\mathrm{Al}$ analizarse los textos sobre deportes, puede observarse claramente que la Globalización implicó un aparente cambio de enfoque genera una nueva conceptualización del deporte, del deportista y de las formas de enseñanza, que se fundan en un aprendizaje más eficaz de cada una de las prácticas deportivas. Este supuesto giro conceptual se da en concordancia con los cambios que se producen en el mundo, es decir, la entrada al neoliberalismo. Bajo este nuevo enfoque que se generó desde el comienzo de la Globalización la enseñanza deportiva se agilizan, se flexibilizan los mecanismos de enseñanza, pero incrementando la necesidad de obtención de resultados. Lo técnico pasa a ser considerado eficaz, si es aplicado con velocidad, siendo para ello necesario una enseñanza que replique de la manera más parecida posible la situación de competencia. Sin embargo, a pesar de que muchas veces este nuevo enfoque es presentado como una posibilidad que atiende a las particularidades del sujeto, lo analizado hasta ahora permite considerar que, por el contrario, requiere de cierta estandarización y unificación, porque es una concepción orientada hacia la búsqueda de rendimiento, que al mismo tiempo pretende la conformación de un modelo óptimo de enseñanza transferible a todos los casos.

\section{Para Nicolás Parra Bolaños, Claudia Herrera Cárdenas y Albeiro Muñoz Giraldo (2017),}

en la década comprendida entre 1975 y 1985 hubo una explosión masiva en el uso de las telecomunicaciones, ya no sólo a nivel militar, gubernamental y privado, haciendo que la neurociencia se adhiriera con relativa celeridad a todo el desarrollo de las Tecnologías de la Información y la Comunicación (TIC), para poder comenzar a llevar a los hogares [...] contenidos relacionados con el funcionamiento del cerebro y de todo el Sistema Nervioso Central (SNC) (2017, p. 20).

Esto influyó de manera determinante en el campo de la enseñanza deportiva, definiendo el momento en el que el tratamiento del deporte genera una producción teórica a gran escala que, sumado a la masividad que alcanzaron los medios de comunicación y la aparición de las redes sociales, que se transformaron en determinantes para la difusión de estas ideas. En este marco, debe comprenderse que los nuevos presupuestos que rompieron con la idea del Estado asistencial y, por lo tanto, el concepto de lo público, generó nuevas formas de prácticas y nuevos actores sociales, con otras formas de participación en el escenario global, como una reacción o respuesta concreta al supuesto viejo esquema derecha-izquierda de la práctica política, que sin dudas alcanzaron al campo deportivo, lo que confirma a la Globalización como un supuesto político, porque pone en escena al mundo empresarial y a sus asociados, posibilitándole un poder negociador inusitado hasta a llegada de este fenómeno. 
La globalización posibilita eso que sin duda estuvo siempre presente en el capitalismo, pero que se mantuvo en estado larvado durante la fase de su domesticación por la sociedad estatal y democrática: que los empresarios, sobre todo los que se mueven a nivel planetario, puedan desempeñar un papel clave en la configuración no sólo de la economía, sino también de la sociedad en su conjunto, aun cuando sólo fuera por el poder que tienen para privar a la sociedad de sus recursos materiales (capital, impuestos, puestos de trabajo) (Beck, 1998, p. 38).

Desde esta nueva óptica, los profesores y entrenadores proclaman tomar como punto central de la enseñanza a las situaciones de juego. El interés y la atención de la misma, ya no se concentra en las acciones, sino en la situación y, en esta coyuntura, la estimulación del cerebro y del Sistema Nervioso Central [en adelante $\mathrm{SNC}$ ], pasaron a ser fundamentales para la reproducción de deportistas más acordes con los avances científicos tecnológicos. Los textos analizados demuestran que, específicamente se busca reproducir en las sesiones de entrenamiento y en las clases determinadas situaciones que habitualmente se generan en un espacio del campo de juego, o de la competencia, para poder planificar las formas más óptimas de resolución de las mismas, lo que determinó un deportista con mayor desarrollo de la atención, la decisión y la reacción a los estímulos (Hours, 2014).

Nicolás Parra Bolaños, Claudia Herrera Cárdenas y Albeiro Muñoz Giraldo (2017) aseguran también que, con la llegada de la Globalización, de manera general, las diversas poblaciones

tuvieron acceso por vez primera a los conocimientos generados en ciencia básica y aplicada, desarrollados por los neurocientíficos de las principales universidades del mundo, logrando así dar un paso hacia adelante con relación a la expansión de las Neurociencias en campos como los sistemas audiovisuales, la publicidad, el mercadeo y, sobretodo, conseguir calarse en el lenguaje de la gente que, atraída por las potentes imágenes que empleó y continua empleando la neurociencia, se ha vinculado a la disciplina y la ha hecho parte de sus vidas $(2017$, p. 21$)$.

Siguiendo esta idea, nuestros análisis demuestran que de manera general, la matriz de todos los estudios que ha ido configurando el campo, tanto del desarrollo deportivo como de su enseñanza, desde la Globalización, se basan en estudios claramente identificables con la Biología y con el desarrollo de lo que en nuestros días se denomina Medicina del Deporte. Desde el enfoque planteado, se puede afirmar entonces que, la Globalización implicó un resurgimiento de las Neurociencias como forma de pensar, planificar e instituir la práctica deportiva en todos sus niveles, porque la funcionalidad orgánica optimizada al máximo pasa a ser el núcleo central del rendimiento. En esta nueva configuración teórico-científica, los trabajos comienzan a pensar en la estimulación del SNC como posibilidad del aumento de rendimiento, tanto técnico como motor, al ritmo de la revolución comunicacional que la Globalización generó en la producción económica. Es así que puede observarse claramente que los textos retoman con mayor énfasis los postulados científico-médicos que regulan el orden que se debe seguir para un correcto progreso en el campo, sobre todo de la enseñanza y en especial desde la niñez. Momento en la vida del ser humano que es clave para la estructuración y eficacia de este proceso planteado por esta forma de pensar la enseñanza y la práctica deportiva.

En este punto nuestra investigación permite que se podamos afirmar que, al igual que en el resto de las actividades sociales, el campo deportivo, de la mano de los argumentos médicos como forma de conocimiento válido pero también inobjetable y con la colaboración del desarrollo tecnológico, fue cobrando mayor importancia el rol de las empresas y la libertad de ideas que acrecienten el ingreso y circulación de capitales, junto con la implantación definitiva de la sociedad de consumo en el campo deportivo. Esta idea para nosotros es central para comprender los avances de las Neurociencias en el plano educativo durante el neoliberalismo, pues define al sujeto preparado para el consumo desde supuestos neurofisiológicos. En nuestra perspectiva el sujeto es definido por las Neurociencias como una forma de prepararlo para su deseo de consumo. Es así que los deportes pasan a ser más que un simple divertimento, para ser un macro-negocio y una forma de normalizar a la población con fines particulares movilizados por la búsqueda de ganancias económicas a gran escala.

El aumento en la cantidad de personas para realizar prácticas deportivas, fue generado en parte por el discurso médico sobre los beneficios que esta acarrea, instalándose ideas que han tendido a universalizar y 
a generalizar la práctica, por ejemplo, que el deporte es un agente beneficioso para salud. Este orden de cosas se corresponde con ciertas normas formales y simbólicas establecidos por la Modernidad, que determinó que, al considerar lo humano, se termine considerando lo animal que hay en él; cuestión que implica por lo tanto, no pensar en el sujeto -aunque se lo nombre permanentemente-, para dar clara preeminencia al individuo. De esta manera, el ser bumano pasa a ser pensado como una especie animal más entre todas las que existen, y de esto existen numerosos argumentos al respecto. Todo esto que es juzgado por las tendencias pedagógicas más actuales, las que también colaboran para sustentar a la ciencia moderna, no es más que una aplicación indiscriminada de un dogma pseudocientífico biológico, que expresado como un avance de la ciencia en relación al ser humano, pocos se atreven a discutir en la actualidad. En este sentido, son muy valiosos los aportes de Merlin (2017), ya que asegura que esto ocurre porque "el sistema capitalista en su variante neoliberal funciona imponiendo ideas a través de los medios de comunicación corporativos y el marketing, que se incorporan, se demandan y terminan naturalizándose. Se trata de un proyecto colonizador que necesita realizar una producción biopolítica de subjetividad, y con ese objetivo se apropia de sentidos y representaciones de la cultura" (Merlin, 2017, s/p).

Habiendo instaurado una lógica mucho más dinámica de funcionamiento que acrecentó y optimizó las posibilidades de resultados favorables a sus fines, con una comunión perfecta, neoliberalismo y Neurociencias se dan la mano para establecer estructuras sólidas que le garantice al mercado, no sólo la producción de ganancias, sino la reproducción de lógicas que aseguren su continuidad y su funcionalidad a futuro, bajo la promesa del desarrollo humano y social. Desde una perspectiva crítica, se puede señalar que la pretendida similitud entre el hombre y los animales, que por momentos parece ser reducida de manera teórica por ciertas ideas sociales o pedagógicas sobre el deporte, retomando las bases neurocientíficas, en realidad termina por exaltar, exacerbar y legitimar lo animal del ser humano en todos sus órdenes. Es así que se puede observar que, al resaltarse las cualidades humanas y sus posibilidades neurofuncionales, no se hace más que individualizar al sujeto, cuestión que concuerda con la idea de Raumar Rodríguez Giménez (2015), quien señala que "la revolución de la ciencia moderna consistió en 'referir conocimiento y experiencia a un sujeto único"' (2015, p. 19), capaz de ser normalizado, formateado y programado.

\section{Conclusiones}

Si bien para Mendoza Gil "visiones prospectivas del fenómeno de la globalización lo ubican como algo novedoso y más aparejado a los tiempos recientes” (2016, s/p), los estudios realizados para esta investigación, demuestran que sus discursos asumen una mirada histórica, que fue desarrollada a niveles superlativos, pero que nada tiene de novedoso, un claro ejemplo de ello es que retoma los estudios de las Neurociencias, que ya desde el siglo XVII se venían desarrollando en Europa como gran interés. Sin embargo, es necesario señalar que, lo cierto es que la Globalización generó cambios en todos los niveles de la sociedad, condicionando de manera similar a la enseñanza deportiva como lo hizo con todas las otras dimensiones de que comprenden la vida humana, estableciendo una teoría más totalizada, universal y general en el campo. Se hace referencia a la concepción natural de la vida humana, porque, de manera general, esta es la idea que sostiene todas sus principios constitutivos, que a la vez más réditos le ha dado; ese pensamiento funcionalista-natural que gobierna el desarrolló del sujeto en estos tiempos, para todos sus órdenes. En el plano de lo deportivo, en materia teórica, se puede concluir que en este período se desarrolló una teoría mucho más elaborada sobre el rendimiento deportivo, que definió con más certezas aun la dimensión animal de lo humano. Como se señaló anteriormente, implicó romper con el sujeto, para dar paso a un individuo que puede cuantificar sus conductas y ser formados a niveles óptimos en términos de rendimiento. Este desarrolló teórico se dio en especial, por los aportes de esa ciencia experimental moderna, que facilitó la objetivación del sujeto. En este sentido, se pudo observar en la mayoría de los textos publicados más recientemente, un rebrote significativo de las neurociencias como soporte teórico del desarrollo deportivo, por lo que podemos señalar que, con una 
comunión perfecta, neoliberalismo y neurociencias se dan la mano para establecer estructuras sólidas que le garantice al mercado, no sólo la producción de ganancias, sino la reproducción de lógicas que aseguren su continuidad y su funcionalidad a futuro, especialmente desde la formación del deportista y desde la legitimación del campo deportivo, como uno científico natural. Esto apoyado en la revolución tecnológica y comunicacional que este proceso implicó e implica, en el que:

"El auge del intercambio de flujos informativos producto de la masificación en el uso de la internet, la telefonía celular y televisión por suscripción, para definir los mecanismos de expansión del proceso globalizador debido a la capacidad para intercambiar y hacer llegar información a los sitios más apartados, en tiempo real, en cualquier lugar de la a geografía mundial, información que lejos de resaltar las particularidades propias de un mundo donde prima la heterogeneidad, pretenden establecer una occidentalización progresiva donde 'lo realmente valido es sólo lo moderno y lo Moderno es sólo lo occidental"' (2016, s/p).

Por esta razón, es necesario considerar, como se señaló anteriormente, que, al igual que en los comienzos de la Modernidad, el campo teórico del deporte refiere su conocimiento y experiencia a un sujeto único, capaz de ser normalizado, formateado y programado al extremo de sus posibilidades. Es así que la revisión teórica del campo deportivo demuestra que en este terreno, la Globalización, entendida como la continuidad histórica lógica y consecuente del capitalismo, no dejó de apuntar a la esfera económica, tratando de imponer el imperio del libre mercado a nivel mundial, “como modelo de organización económica y aspectos más amplios sustentados en la promoción de la democracia como doctrina política y la occidentalización cultural” (ídem, $\mathrm{s} / \mathrm{p}$ ), ocasionando una ruptura como lo autóctono de las sociedades menos dominantes, hacia una tendencia impuesta por las sociedades dominantes. En este orden, se puede afirmar que, en la afirmación de lo natural y la ausencia o nula alusión a lo político, que se presenta en cada argumento que estructura la teoría deportiva, existe una posición política que claramente se vincula con la productividad del sujeto, el mercado y el sistema económico, desde una base eminentemente biológica y médica que dota de cierto cientificismo su supuesto saber.

Por último, algo sumamente relevante que arrojo la investigación, que ha alcanzado en especial al campo de la Educación Física, es que estas tendencias supuestamente más desarrolladas del tratamiento deportivo en el marco de la Globalización concuerdan "con una visión complementaria de entender la cultura deportiva, que conjuguen o converjan a los científicos y tecnólogos por un lado, con los humanistas y creadores por otro" (ídem, s/p). Bajo esta perspectiva que complementa lo natural con lo humanista, los textos asumen la promoción de discursos que apuntan a la inclusión de mayor cantidad de personas que se inserten hacia la práctica deportiva, en general, transmitiendo dicha práctica como una necesidad natural en el sujeto y, por lo tanto, necesaria para la población y la sociedad.

\section{REFERENCIAS BIBLIOGRÁFICAS}

Almonacid, A. (2012). La educación física como espacio de transformación social y educativa: perspectivas desde los imaginarios sociales y la ciencia de la motricidad humana. Revista Estudios Pedagógicos, 38(1), 177-190.

Beck, U. (1998). ¿Qué es la globalización? Falacias del globalismo, respuestas a la globalización. Barcelona: Ediciones PAIDÓS.

Dos Santos, S.; Crestani, E., Montenegro, J. y Lara L. (2015). Dimensiones de la educación física escolar colombiana e incursiones por la tematización de la cultura y la calidad en la educación. Revista de Investigación Cuerpo, Cultura $y$ Movimiento, 5(1), 35-56.

Dunning, E. (2009). Reflexiones sociológicas figurativas y de proceso sobre el deporte y la globalización: algunas observaciones conceptuales y teóricas, con especial referencia al fútbol. Apunts Educación Física y Deportes, 97, 8-17.

Hours, G. (2014). Los discursos de la enseñanza deportiva. Mitos, tradiciones y naturalizaciones. Análisis del discurso de la Iniciación Deportiva española; Tesis para optar al grado de Magister en Deporte, Maestría en Deporte, Facultad 
de Humanidades y Ciencias de la Educación, Universidad Nacional de La Plata, http://hdl.handle.net/10915 134673 .

Lara, L. (2019). Educação Física e Cultura na América Latina. Maringá: Editora da Universidade Estadual de Maringá.

Mendoza Gil, W. (2016). Deporte globalizado/Deporte en la globalización. La continuidad y discontinuidad del deporte profesional capitalista: realidades y perspectivas. Espacio Abierto. Cuaderno Venezolano de Sociología, $25(2)$

Merlin, N. (2017). Colonización de la subjetividad: las Neurociencias.La Tecl@Eñe.Revista de Cultura y Politica, 15 de marzo. https://lateclaenerevista.com/ [Fecha de consulta: 23/10/2019].

Parra Bolaños, N.; Herrera Cárdenas, C. y Muñoz Giraldo, A. (2017). Las Neurociencias como herramienta de comunicación y desarrollo de los procesos de globalización. Revista Chilena Neuropsicología, 12(1), 19-23. http s://10.5839/rcnp.2017.12.01.03.

Rocha Bidegain, L. (2017). Educación física o educación corporal. Biologismo, nihilismo, individualismo. En S. Achucarro, N. Hernández, y D. Di Domizio (Comps.), Educación física: Teorías y prácticas para los procesos de inclusión. La Plata: Universidad Nacional de La Plata Facultad de Humanidades y Ciencias de la Educación. (Colectiva y monográfica ; 3). Recuperado de http://libros.fahce.unlp.edu.ar/index.php/libros/catalog/book/ 92

Rodríguez Giménez, R. (2015). La vida en la encrucijada de lo humano (o lo pedagógico y el hombre en tanto que “vive". Revista del Departamento de Pedagogía, Politica y Sociedad del Instituto de Educación de la FHUCE, Pedagógica, 3, marzo.

Vélez de Fonnegra, M. (2011). Neurociencias y educación. Recorriendo sendas paralelas. Curso de Capacitación Docente en Neurociencias. Inédito. Disponible en: https://asociacioneducar.com/monografias-docente-Neuro ciencias/velez.pdf. [Fecha de consulta: 12/05/2020].

\section{Notas}

1 Hours, G. (2018). Director. Los discursos de la enseñanza de los deportes desde la perspectiva de la educación física. Análisis sobre la construcción teórica en el campo desde el comienzo de la globalización / H047. (Proyecto de investigación). UNLP. FaHCE. Área de Estudios e Investigaciones en Educación Física. Disponible en: http://www.memoria.fahce.unlp.edu .ar/proyectos/py.766/py.766.pdf.

2 Rocha Bidegain, L. (2019) Directora. Prácticas corporales y educación del cuerpo: el discurso neurocientífico como imposible para la enseñanza / H896. Desde 01-01-2019 hasta 31-12-2023. 